AMERICAN JOURNAL OF SOCIAL AND MANAGEMENT SCIENCES

ISSN Print: 2156-1540, ISSN Online: 2151-1559, doi:10.5251/ajsms.2010.1.1.55.66

(C) 2010, ScienceHuß, http://www.scihub.org/AJSMS

\title{
Issues in relation to discounted cash flow valuation
}

\author{
J. P. Singh and Shigufta Hena Uzma \\ Department of Management Studies \\ Indian Institute of Technology Roorkee, Roorkee 247667, Uttarakhand, \\ jatinfdm@iitr.ernet.in, jpsiitr@gmail.com \\ Research Scholar, Department of Management Studies \\ Indian Institute of Technology Roorkee, Roorkee 247667, Uttarakhand \\ suzmaddm@iitr.ernet.in
}

\begin{abstract}
The current era of "convergence through connectivity" is slowly but certainly acknowledging the contribution of the so-called "intangibles" like brands, copyrights \& patents, human \& intellectual capital etc. to the bottomlines of companies. As an obvious corollary, issues relating to the valuation of such assets are surfacing with unprecedented regularity. Valuation of such assets posits an intriguing challenge for the accounting fraternity that is entrenched in the traditional ascendancy of "reliability" over "relevance". "Discounted Cash Flow" is ubiquitous in financial valuation. In fact, this technique constitutes the cornerstone of contemporary valuation theory. The robustness of the model as well as its compatibility with the conventional two dimensional risk-return framework of investment appraisal make it immensely suited to a multitude of asset/liability valuations. Accounting standards across the globe recognize the efficacy of this model and advocate its use, wherever practicable. FAS 141 \& 142 of the United States \& IAS 39 that relate to the accounting of intangible assets also recommend use of DCF methodology for imputing a value to such assets. FAS 157 read with Concept Paper 7 mandate its use for ascertaining "fair value" of assets in certain cases. It is pertinent to note that the usual option pricing methods (including Black Scholes) also make use of discounted cash flows for calculating instantaneous option premia. However, like all models, DCF is not without its flaws. The model presupposes the existence of several unrealistic and rigid assumptions including, in particular, the existence of an acceptable "measure of risk" which is such that it can be integrated with the "discount" rate. In this article, we attempt to address all these issues. We start by highlighting the versatility of the DCF technique, how it can be adapted to value (literally) any asset/investment e.g. securities, projects, corporates and, now, intangibles as well. This enables us to perform a dissection of this model and adduce its anatomy. While on this, we also propose to explore interrelationships of DCF with other extant methods of valuations that include income multipliers, residual income, accrual accounting based methods etc. In today's world of "fly by night" corporate operators, it is paramount to examine the susceptibility of a model to manipulations, and we also examine this facet of the DCF model by conducting a comprehensive sensitivity analysis with respect to the input variables that include estimated/projected cash flows, discount rates, horizon values, the existence of abandonment options etc. As is inevitable, the DCF methodology has its own spectrum of limitations. While concluding this article, we present some of the important shortcomings of this model and make recommendations on its possible upgradations to enhance its efficacy and reliability.
\end{abstract}

\section{INTRODUCTION}

"Discounted Cash Flow" is ubiquitous insofar as asset valuation goes with the method possessing the flexibility, adaptability and robustness to value literally, at least in theory, any asset under the sun, be it a security, project, corporate or an intangible or any combination thereof. In fact, the nexus between "Discounted Cash Flow" and valuation is so proximate that practitioners have ascribed a distinct identity to the valuation so obtained as "intrinsic" value. Its compatibility with the conventional two dimensional risk-return framework of investment appraisal makes it immensely suited to a multitude of valuation exercises. Accounting standards across the 
globe recognize the efficacy of this model and advocate its use, wherever practicable. FAS 141 \& 142 of the United States \& IAS 39 that relate to the accounting of intangible assets also recommend use of DCF methodology for imputing a value to such assets. FAS 157 read with Concept Paper 7 of the United States mandate its use for ascertaining "fair value" of assets in certain cases. It is pertinent to note that the usual option pricing methods (including Black Scholes) also make use of discounted cash flows for calculating instantaneous option premia.

The elegance of the method lies in its perceived simplicity - one merely projects the anticipated cash flows from the asset and estimates the return that may be desired commensurate with the risk profile of the projected cash flows and the asset value is spontaneous. Stated symbolically,

$$
\text { Intrinsic Value }=\sum_{i=0}^{n} C_{i} e^{-r_{i} t_{i}}
$$

where $C_{i}$ is the $i^{\text {th }}$ cash flow occurring at time instant $t_{i}$ of a series of $n$ cash flows from the asset and $r_{i}$ is the annualized interest rate (with continuous compounding) corresponding to a maturity of $t_{i}$ (which is measured in years).

However, God could surely not have made "money matters" so uncomplicated and undemanding and, indeed, it is not so - while the computation of intrinsic value is the conventional "child's play", the estimation of inputs to the model is beset with numerous complexities and nuances and it is at this point that the professional's wisdom is truly tested. Not only does one face the intrinsic vagaries of "future" in making projections of inputs but one needs also to address the equally vital issue of identifying the appropriate inputs compatible with the "valuation" exercise envisaged. Needless to say, the nature of the inputs would vary with the type of asset to be valued.

The bottomline is that DCF is also a model and like all models it cannot simulate "reality" to exactness. Were it to be otherwise, then the model would also encompass the evolution equations of "reality" enabling it to project reality, which is surely not the case.
DCF is not without its flaws. The model presupposes the existence of several unrealistic and rigid assumptions including, in particular, the existence of an acceptable "measure of risk" which is such that it can be integrated with the "discount" rate. In this article, we attempt to address all these issues. In Section 2, we highlight the versatility of the DCF technique by elucidating its adaptability to value securities, projects, corporates and intangibles as well. This enables us to perform a dissection of this model and adduce its anatomy. While on this, we explore interrelationships of DCF with other extant methods of valuations that include income multipliers, residual income, accrual accounting based methods etc. In today's world of "fly by night" corporate operators, it is paramount to examine the susceptibility of a model to manipulations. We also examine this facet of the DCF model by conducting a comprehensive sensitivity analysis with respect to the input variables that include estimated/projected cash flows, discount rates, horizon values, the existence of abandonment options etc. As is inevitable, the DCF methodology, has its own spectrum of limitations. While concluding this article in Section 3, we present some of the important shortcomings of this model and make recommendations on its possible upgradations to enhance its efficacy and reliability.

\section{DCF \& Asset Valuation}

Convexity of the Yield-Price Curve (Elton, 1997, Fabozzi, 2002, Golub, 2000, Tuckman, 2002)

Valuation of plain vanilla fixed income securities is widely perceived as the simplest valuation exercise, probably because the cash flows from these assets are, usually, fixed by the terms of issue and are contractual in nature. The discount factors are the spot interest rates that can be read off from the prevalent yield curve. However, notwithstanding this naïve computational procedure, investment decisions taken on this premises alone, are likely, more often than not, to be sub-optimal on several counts.

First and foremost, the relationship between price and yield is not a linear one (for interest/discount rates with continuous compounding, it becomes a sum of exponentials e.g. eq. (1)). This has several intriguing repercussions. To keep the theme tractable and not lose it in a plethora of calculations at this stage, we assume the yield curve to be flat i.e. that the interest rates are independent of maturities, 
so that eq. (1) becomes $P=\sum_{i=1}^{n} C_{i} e^{-r t_{i}}$. The first derivative (that defines the slope of the yield-price curve) gives $\frac{d P}{d r}=-\sum_{i=1}^{n} t_{i} C_{i} e^{-r t_{i}}$. The slope is, obviously negative at all points with the magnitude thereof decreasing with increasing yields, thereby establishing the well known "convexity" of the yieldprice curve. What are the implications for the investment manager? There are several (Salomon, 1995) e.g.

(a) The bond's price rise for a given fall in yield is greater than its fall for an equal rise in yield. This is easily seen by considering a zero coupon bond of unit redemption value and maturity, say $T$ years. Its current market price corresponding to a yield $y$ is given by $P(y)=e^{-y T}$. Let $\pm d y$ be the possible changes in the instantaneous yield in either direction of the current yield. Then, expanding the price function as a Taylor's series around $y$, we get

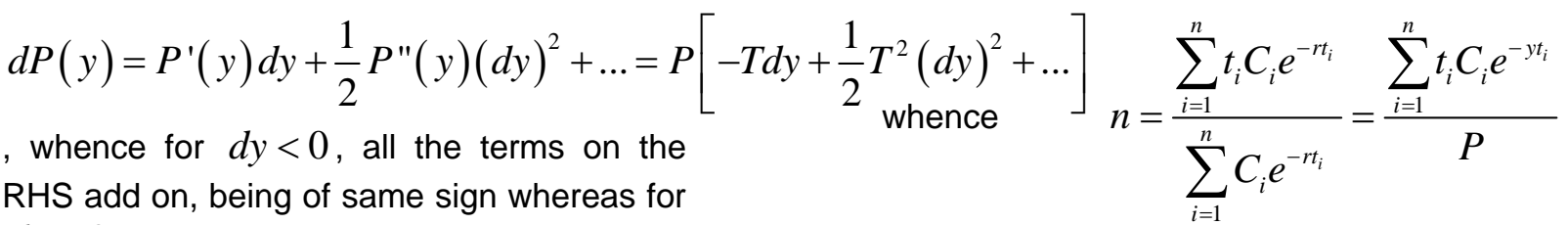
$d y>0$, the positive and negative terms setoff to some extent (that depends on the current yield $y$ ). It follows that if $\pm d y$ are weighted with equal probability i.e. that the yield curve changes have a symmetric probability distribution around $y$, then, the expected price shall exceed the current price $P(y) \quad$ because $E(P)=0.5[P(y+d y)+P(y-d y)]=$ $P\left[1+\frac{1}{2} T^{2}(d y)^{2}+\ldots\right]>P . \quad$ It, follows, then, that investors investing in fixed income securities in ignorance of the possible direction of movement of interest rates should expect gains to exceed losses or, in other words, expect a positive return provided, of course, that the interest rates follows a symmetrical distribution in the region of relevance. Not only this, the more convex the yield-price curve is, the greater would be the differential between expected returns and realized yields.

Another fallout of the above is that there does, definitely, exist a relationship between the volatility of interest rates and the enhancement effect on expected returns of the convexity of the yield-price curve.

(b) There exists a unique holding period corresponding to each point on the yieldprice curve, such that if the bond is held for the said period, the total cash inflows emanating from the bond become insensitive to infinitesimal changes in interest rates around the given point. This holding period is the so called "duration" of the bond. An expression for the "duration" is obtained from the total cash flows, TCF , emanating from the security for a holding period of $n$ years as

$0=\frac{d(T C F)}{d r}=\frac{d}{d r} \sum_{i=1}^{n} C_{i} e^{r\left(n-t_{i}\right)}=\sum_{i=1}^{n}\left(n-t_{i}\right) C_{i} e^{r\left(n-t_{i}\right)}$ where $y$ is the yield to maturity. We also have, $\frac{d P}{d r}=-\sum_{i=0}^{n} t_{i} C_{i} e^{-y t_{i}}=-n P$ whence we can also interpret "duration" as a measure of the price sensitivity of the bond to changes in yields.

At this point, it needs to be emphasized that due to the convexity of the yield-price curve, the immunization afforded by "duration" is only for infinitesimal interest rate changes in the neighbourhood of the rate that has been adopted for computation thereof - when there is a significant "duration" are bound to be inexact with the error escalating with the magnitude of the change in yield.

Mathematically, calculations of price differentials corresponding to interest rate changes based solely on the "duration" of a bond assume a linear yieldchange in yield, price variations calculated using 
price relationship in the area of interest i.e. we retain only the first degree terms in $d y$ in the Taylor's series expansion of $P(y)$. Hence, because the curve is convex, the validity of such computations is confined merely to infinitesimals. Practitioners often enhance the accuracy of this frame work by truncating the Taylor's series beyond the second degree terms instead of first degree terms in $d y$ whence, we may write

$d P(y)=P^{\prime}(y) d y+\frac{1}{2} P^{\prime \prime}(y)(d y)^{2}=-n P d y+c P(d y)^{2}$

where $c=\frac{\sum_{i=1}^{n} t_{i}^{2} C_{i} e^{-r t_{i}}}{2 P}=\frac{1}{2 P} \frac{d^{2} P}{d y^{2}}$ is usually termed as the "convexity" of the bond and $n=\frac{\sum_{i=1}^{n} t_{i} C_{i} e^{-r t_{i}}}{P}=-\frac{1}{P} \frac{d P}{d y}$ is the above referred "duration".

The convexity of a bond plays a significant role in bond portfolio management. Since the linear approximation implicit in "duration" based computation always lies below the curve in the case of curves with positive convexity, such approximation always understates a bond's price change for a change in yield. Advent of convexity reduces this error.

All conventional bonds have positive convexity. In fact, negative convexity occurs occasionally in case of bonds that have attached option features. Even in such cases, the negative convexity occurs only for a certain range of interest rates because the existence of the call option effectively curtails the bond's price appreciation potential. For significant decline in interest rates, the issuer of such bonds would find it profitable to redeem the existing bonds and replace them with debt at current rates (which is lower) thereby optimizing cost of funds.

Convexity and duration are intimately related for $2 c P=\frac{d^{2} P}{d y^{2}}=\frac{d}{d y}(-n P)=-\frac{d n}{d y} \cdot P-n \frac{d P}{d y}=-\frac{d n}{d y} \cdot P-n(-n P)$ so that $c=\frac{1}{2}\left(-\frac{d n}{d y}+n^{2}\right)$. It is pertinent here to elaborate the significance of the first term. It measures the rate of change of duration with respect to the yield. Now, the quantum and incidence of cash flows of most fixed income securities is fixed. $\frac{d n}{d y}$ would, therefore, essentially depend on the spread or pattern of these cash flows i.e. the cash flow dispersion. For instance, $\frac{d n}{d y}$ for zero coupon bonds would be zero and would increase with the distribution of coupon payments.

$$
\begin{aligned}
& \text { "Duration" and "Convexity", because of their } \\
& \text { interpretation as the price sensitivity } \\
& \text { indicators of the underlying security to yield } \\
& \text { rate changes, enable a very powerful and } \\
& \text { versatile framework for interest rate } \\
& \text { management that includes hedging against } \\
& \text { interest rate fluctuations and constitute } \\
& \text { widely adopted tools of asset-liability } \\
& \text { management by banks and financial } \\
& \text { institutions. However, the efficacy of this } \\
& \text { framework presupposes the very strong } \\
& \text { assumption of "parallel" shifts in the yield } \\
& \text { curve. Let us look at this more closely. }
\end{aligned}
$$

As mentioned earlier, duration can be computed at any point on the yield-price curve i.e. with respect to any interest rate. It, then, provides immunity against infinitesimal movements of interest rates around that particular rate. Such infinitesimal movements of interest rates correspond to infinitesimal pulses or shocks in the yield curve at the relevant point. Now, let us consider a slightly modified scenario e.g. the "yield based duration" hedging of a 6 year liability by a 1 year and 10 year assets portfolio. The hedging would be efficacious only in the event that the magnitude and direction of the infinitesimal interest rate change are identical across the spectrum of both, the hedged liability and the hedging asset. This implies that the entire yield curve shifts infinitesimally but parallel to the original curve at all points or equivalently, that the underlying factor that causes the interest rate change affects rates of all maturities to the same extent e.g. the change brought about by some underlying factor is the same, be it the six month rate or the sixty year rate. This is a bizarre assumption and certainly not supported by empirical data. As an approach to obviate this problem to some extent, practitioners sometimes take recourse to the concept of "key rates". The methodology essentially lies in identifying a set of maturities such that the corresponding interest rates are able to, through 
mutual interaction, if necessary, model the entire yield curve i.e. given the changes in values of the key rates, the model enables the determination of the changes in interest rate of any maturity. The type of such rates, their number, domain of influence and the nature of mutual interaction is left to the judgement of the analyst. Some conventions in this regard are, however, prevalent among financial practitioners e.g. (i) the domain of influence of each key rate extends from the term of the previous key rate (or zero) to the term of the next key rate (or the last rate); (ii) at its own maturity, the influence of a key rate change is $100 \%$ of the change and this influence declines linearly on either side to zero at the term corresponding to the adjacent key rates. However, in the region between zero and the first key rate and that between the last key rate and the last rate, the influence remains constant at $100 \%$.

Thus, the price of a security becomes a function of these key rates i.e. $P=P\left(r_{1}, r_{2}, r_{3}, \ldots, r_{m}\right)$. We, can, again, define "key rate durations" as measures of price sensitivity i.e. $n_{r_{i}}=-\frac{1}{P} \frac{\partial P}{\partial r_{i}}$ and represent a "price change" due to changes in all the key rates as the aggregate $\frac{\Delta P}{P}=-\sum_{i=1}^{m} n_{r_{i}} \Delta r_{i}$. The interest rate hedging strategy would then take the form of "key rate wise duration" matching of the liability and assets and would, at least in theory, provide effective immunization against interest rate shifts.

\section{Stochasticity of market interest rates (Singh, 2006)}

In the above analysis, the interest rate $r$ is taken as constant. However, this rate would, nevertheless, be subject to uncertainties that influence returns on the fixed income securities. It is, now, conventional to model these interest rates through a stochastic differential equation of the form (Wilmott, 2000)

$$
d r(t)=-\psi[r(t), t] d t+\eta[r(t), t] d U(t)
$$

where $r(t)$ is the interest rate at time $t, \psi$ and $\eta$ are deterministic functions of $r, t$ and $U(t)$ is a Wiener Process.

In our further analysis, we shall assume that this interest rate is represented by the Vasicek model (Vasicek, 1977) viz. $\frac{d r(t)}{d t}+A r(t)+B-\sum \eta(t)=0$

where $\eta(t)$ is a white noise stochastic process (Revuz, 1999)

$\langle\eta(t)\rangle=0,\left\langle\eta(t) \eta\left(t^{\prime}\right)\right\rangle=\Sigma^{2} \delta\left(t-t^{\prime}\right)$

We can, now, obtain explicit expression for the discount factor $I=\left\langle e^{-\int_{0}^{t} r(\tau) d \tau}\right\rangle$ averaged over the stochastic part of the interest rate process. To evaluate this expectation integral $I$ we make use of the functional integral formalism. In this formalism, the expectation $I$ would be given by (Feynmann, 1965, Martin, 1973):-

$I=\frac{\int_{r(0)}^{r(t)} \operatorname{Drexp}\left[-\frac{1}{2 \Sigma^{2}} \int_{0}^{t} d \tau\left(\frac{d r(\tau)}{d \tau}+\operatorname{Ar}(\tau)+B\right)^{2}-\int_{0}^{t} d \tau r(\tau)\right]}{\int_{r(0)}^{r(t)} \operatorname{Drexp}\left[-\frac{1}{2 \Sigma^{2}} \int_{0}^{t} d \tau\left(\frac{d r(\tau)}{d \tau}+A r(\tau)+B\right)^{2}\right]}=\frac{P}{Q}$

where $D r=\prod_{\tau=t}^{T} \frac{d r(\tau)}{\sqrt{2 \pi}}$ is the functional integration measure.

We first evaluate the functional integral $P$. Making the substitution $x(\tau)=-\frac{B}{A}-r(\tau)$, we obtain, with a little algebra,

$P=\int_{x(0)}^{x(t)} D x \exp \left[-\frac{1}{2 \sum^{2}} \int_{0}^{t} d \tau\left(\frac{d x(\tau)}{d \tau}+A x(\tau)\right)^{2}-\int_{0}^{t} d \tau\left(-\frac{B}{A}-x(\tau)\right)\right]$ $=\int_{x 0}^{(t)} \operatorname{Dexp}\left\{\frac{-1}{2 \Sigma^{2}} \int_{0} d t\left[\left(\frac{d(\tau)}{d \tau}\right)^{2}+A^{2} x^{2}(\tau)\right] \frac{A}{2 \Sigma^{2}}\left[x^{2}(t)-x^{2}(0)\right]+\frac{B(t)}{A[t}+\int_{0}^{t} x(\tau) d \tau\right\}$

$=\int_{x(0)}^{x(t)} D x \exp \left\{-\frac{A}{2 \sum^{2}}\left[x^{2}(t)-x^{2}(0)\right]+\frac{B(t)}{A}-I_{1}\right\}$

where

$I_{1}=\frac{1}{2 \sum^{2}} \int_{0}^{t} d \tau\left[\left(\frac{d x(\tau)}{d \tau}\right)^{2}+A^{2} x^{2}(\tau)\right]-\int_{0}^{t} x(\tau) d \tau$ 
$=\frac{1}{2 \Sigma^{2}} \int_{0}^{t} d \tau\left[\left(\frac{d x(\tau)}{d \tau}\right)^{2}+A^{2} x^{2}(\tau)-2 \sum^{2} x(\tau)\right]$

In order to evaluate $I_{1}$, we perform a shift of the functional variable $x(\tau)$ by some fixed function $y(\tau)$ i.e. $x(\tau)=y(\tau)+z(\tau)$ where $y(\tau)$ is a fixed functional (whose explicit form shall be defined later) but with boundary conditions $y(0)=x(0), y(t)=x(t)$ so that $z(\tau)$, then, has Drichlet boundary conditions i.e. $z(0)=z(t)=0$.

Substituting $x(\tau)=y(\tau)+z(\tau)$ in (7), we obtain $I_{1}=\frac{1}{2 \Sigma^{2}} \int_{0}^{t}\left[\begin{array}{l}d \tau\left[\frac{d y(\tau)}{d \tau}\right)^{2}+\left(\frac{d z(\tau)}{d \tau}\right)^{2}+2\left(\frac{d y(\tau)}{d \tau}\right)\left(\frac{d z(\tau)}{d \tau}\right)+A^{2} y^{2}(\tau)+ \\ A^{2} z^{2}(\tau)+2 A^{2} y(\tau) z(\tau)-2 \Sigma^{2} y(\tau)-2 \Sigma^{2} z(\tau)\end{array}\right]$ (8) Integrating the second and third term by parts, we get

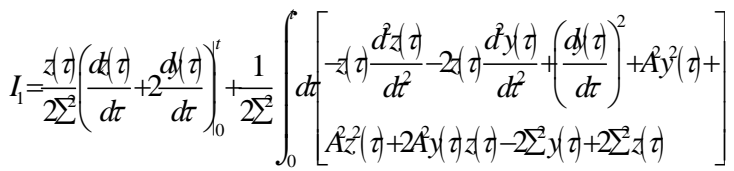

Now the boundary terms all vanish since $z(\tau)$ has Drichlet boundary conditions.

Further, if we define the fixed functional $y(\tau)$ in terms of the differential equation

$-\frac{d^{2} y(\tau)}{d \tau^{2}}+A^{2} y(\tau)+\Sigma^{2}=0$

with boundary condition $y(0)=x(0), y(t)=x(t)$ we obtain

$I_{1}=\frac{1}{2 \Sigma^{2}} \int_{0} d \tau\left\{\left[\left(\frac{d y(\tau)}{d \tau}\right)^{2}+A^{2} y^{2}(\tau)+2 \Sigma^{2} y(\tau)\right]+\left[-z(\tau) \frac{d^{2} z(\tau)}{d \tau^{2}}+A^{2} z^{2}(\tau)\right]\right\}$

The functional $y(\tau)$ is fixed and is given by the solution of eq (10) as

$y=\alpha e^{A \tau}+\beta e^{-A \tau}-\gamma$

where
$\gamma=-\frac{\Sigma^{2}}{A^{2}}, \alpha=\frac{x(t) e^{A t}-x(0)}{e^{2 A t}-1}+\gamma \frac{e^{A t}-1}{e^{2 A t}-1}$

and

Integrating out the $y(\tau)$ terms in eq. (11) using eq. (12), we obtain

$\left.I_{1}=\frac{1}{2 \Sigma^{2}}\left\{A\left[\alpha^{2}\left(e^{2 \beta}-1\right)-\beta^{2}\left(e^{-2 \alpha}-1\right)-A\right)^{2} t\right]+\int_{0}^{t} d \tau\left[-z(\tau) \frac{d^{2} z(\tau)}{d \tau^{2}}+A^{2} z^{2}(\tau)\right]\right\}$

Substituting this value of $I_{1}$ in eq. (7) we obtain, for $P$, noting that $D x=D z$

since $y(\tau)$ is fixed by eq (11)

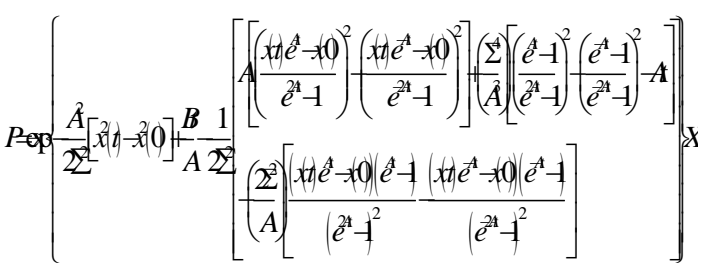

where

$X=\int_{z(0)=0}^{z(t)=0} D z \exp \left\{\frac{-1}{2 \Sigma^{2}} \int_{0}^{t} d \tau\left[-z(\tau) \frac{d^{2} z(\tau)}{d \tau^{2}}+A^{2} z^{2}(\tau)\right]\right\}$

On exactly same lines, we obtain

$\left.Q=\exp \left\{\frac{A^{2}}{2 \Sigma^{2}}\left[x^{2}(t)-x^{2}(0)\right]-\frac{1}{2 \Sigma^{2}}\left[A\left(\frac{x(t) e^{A x}-x(0)}{e^{2 \alpha}-1}\right)^{2}-\left(\frac{x(t) e^{-A}-x(0)}{e^{-2 A}-1}\right)^{2}\right]\right]\right\} X$

Hence

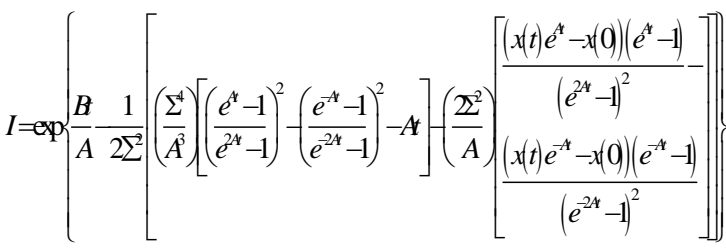

The above procedure shall need to be followed to compute the averaged discount factors corresponding to each cash flow from the asset. This analysis also illustrates how a seemingly innocuous problem has so many intricacies attached with it.

Compatibility of the inputs with the Valuation Exercise 
As has been mentioned earlier, the choice of cash flows as well as the discount rates must be compatible with the valuation exercise envisaged. Again, this seems to be a rather obvious, insipid and an unnecessary assertion. However, the reader would, surely, appreciate the underlying premises by the time he concludes this section. Let us look at corporate valuations by way of a case study. The following methods have been propounded by theorists as possible alternatives for imputing a value to a company (Fernandez, 2007):

(a) Discounting of the projected free cash flows (FCF) of the company at its weighted average cost of capital (WACC) based on the premises that the market value of the company's capital employed (long term debt and equity) is the present value of the firm's projected free cash flows discounted at its WACC i.e.

$$
E(0)+D(0)=\sum_{t} P V(F C F(t), W A C C(t))
$$

For this purpose, we define

(i) "free cash flows" as the residual cash flows of the firm after meeting all operational and investing requirements and other needs for business maintenance including capital expenditure, working capital enhancements less funds received from any funding sources during the period;

$$
W A C C(t)=\frac{E(t-1) k_{e}(t)+D(t-1) k_{d}(t)(1-T)}{E(t-1)+D(t-1)}
$$

(b) Discounting the equity (debt) cash flows at the required return to equity (debt) to obtain the market value of equity (debt) i.e.

$$
\begin{aligned}
& E(0)=\sum_{t} P V\left(\operatorname{EquityCF}(t), k_{e}(t)\right), \\
& D(0)=\sum_{t} P V\left(\operatorname{DebtCF}(t), k_{d}(t)\right)
\end{aligned}
$$

where DebtCF $(t)=I(t)-\Delta D(t)$ i.e. the debt cash flows in a year equal the interest paid in the year as reduced by any debt raised during the year. (c) Discounting the firm's capital cash flows at the firm's pretax WACC so that

$$
E(0)+D(0)=\sum_{t} P V\left(C C F(t), W A C C_{\text {pretax }}(t)\right)
$$

where $C C F(t)$ is defined as the cash flow available to all the company's providers of capital so that

$$
C C F(t)=\operatorname{EquityCF}(t)+\operatorname{DebtCF}(t)
$$

and

$W A C C_{\text {pretax }}(t)=\frac{E(t-1) k_{e}(t)+D(t-1) k_{d}(t)}{E(t-1)+D(t-1)}$

(d) By computing the "Adjusted Present Value" of the firm defined as the value of the unlevered firm plus the present value of tax shields on debt interest. Symbolically,

$$
E(0)+D(0)=E_{\text {unlevered }}(0)+P V T S(0)
$$

with

$$
E_{\text {unlevered }}(0)=\sum_{t} P V\left(F C F(t), k_{e, \text { unleverd }}(t)\right)
$$

. The issue of the "present value of tax shields" is far from settled as of today. Not only is the appropriate discount rate for valuing these shields open to serious debate among academicians, but also the very issue of assigning a value to these shields has been questioned. In an extension of the Miller-Modigliani proposition, Miller (Miller, 1977) has stated that "I argue that even in a world in which interest payments are fully deductible in computing corporate income taxes, the value of the firm, in equilibrium, will still be independent of its capital structure". On the other extreme, the same author, in an earlier work, in co-authorship with Modigliani (Modigliani \& Miller, 1958) has attributed a value to these tax shields (for perpetuity) by discounting the tax savings due to interest payments at the riskfree rate. Myers (Myers, 1974) prefers the discount rate to be reflective of the cost of debt on the premises that the tax savings due to interest payments on debt have the same risk profile as the parent debt. Contrary to this, discounting of 
tax shields is advocated at the unlevered cost of equity by Harris \& Pringle (Harris \& Pringle, 1985). The rationale is that such tax shields have the same systematic risk as the firm's operational cash flows.

(e) Aggregating the current book value of the company's equity and the present value of the Economic Profit discounted at the equity's required rate of return i.e.

$$
E(0)=E_{\text {BookValue }}(0)+\sum_{t} P V\left(E P(t), k_{e}(t)\right)
$$

where "Economic Profit" is the excess of the company's profit after tax over the required return on the company's equity at book value so that

$$
E P(t)=P A T(t)-E_{\text {BookValue }}(t-1) k_{e}(t) \text {. }
$$

Discounting the Economic Value Added at the company's WACC i.e.

$$
\left.E(0)+D(0)=E_{\text {Bakdke }}(0)+D_{\text {Bodkdte }}(0)+\sum_{t} P V(E N A(t) \text {,WACC } t)\right)
$$

Computation of EVA is similar to that of Economic Profit and is equivalent to

$$
E V A(t)=N C P A T(t)-\left[E_{\text {Bodkdue }}(t-1)+D(t-1)\right] * W A C C(t)
$$

This is not the end. There are several other variants of DCF e.g. the discounting of certainty equivalent free cash flows (equity cash flows) at the riskfree rate, discounting of business specific risk adjusted free (equity) cash flows at the required rate of return on assets (equity) etc.

Just like corporate valuations, project evaluation using DCF has its own set of nuances. By way of illustration without, in any way, claiming to be exhaustive, the following are elucidated (Anderson, 2000):

(a) The discount rate (which, in essence, constitutes the "hurdle rate"), must necessarily reflect the market's perception about the return-risk profile of the project. A measure for this is provided by the opportunity cost of the relevant source of funds i.e. the maximum return that the investor can earn by investing in an alternative opportunity with an equivalent risk profile. Furthermore, since the investors realize only the after-tax remnant cash flows from their investments, the entire evaluation framework needs to be administered on an after-tax basis.

(b) While evaluating projects, the "hurdle rate" should relate to the projected costs associated with funds raised for the project, it should not be influenced by historical costs.

(c) The "hurdle rate" should, ideally, encompass all relevant information about the entire market's perceptions about all factors that would influence the project. That would include local i.e. company specific factors, as well as global or industry and economic factors.

(d) There is some rationale in the views propounded by a school of theorists that the "hurdle rate" should be the cost of capital of the firm rather than be a project specific "rate". The logic stems from the fact that there may be certain correlations between the current activities of the firm and the proposed project that may carry synergetic values. Besides, it is, obviously, neither practicable nor justified in treating the project as a completely isolated system.

(e) The cardinal fallout of the CAPM is that market does not reward investors for "unsystematic risk" on the premises that (i) such risk can and should be eliminated by appropriate diversification and (ii) the market participants have adequate expertise to achieve such elimination. This logic carries over to projects as well, for, as mentioned above, such appraisal needs to ultimately manifest market's outlook about the project rather than the management's perceptions. It follows that investors would not allocate value for any risk that is perceived by them as "unnecessary". Thus, the "hurdle rate" needs to be in tandem with the mandatory risks that emanate from the envisaged project. Now, this throws up several intricate and, possibly, unresolved issues as per the current state of knowledge e.g. how to measure project risk, how to segregate such risk as being "necessary" or otherwise and, most importantly, how to map such risk to return rates i.e. how to evolve an appropriate risk-return trade off compatible with the investor community. 
The impact of "convexity" of the yield-price curve on the pricing of fixed income securities has already been addressed above. The impact of convexity carries over to all discounting problems. It is, however, lost sight of, in most cases. Nevertheless, such convexity implies that discounting of cash flows across time using the same rate results in increased discounting for farther periods than implied by a linear discounting structure using the same discount rate.

In line with the "conservational" attitude of accountants, it is appropriate to discount risky inflows with a relatively higher rate and conversely, risky outflows with a lower rate. The logic is simple viz. the higher the risk associated with an outflow, the greater should be its impact (adverse, due to uncertainty) on the project which mandates that we use a lower discount rate to assign a relatively higher value to the outflows. In fact, this principle has universal validity and if there are individual cash flows in the bucket with widely differing risk characteristics, such cash flows should be discounted as separate streams with risk-compatible discount rates.

(h) Evaluation of projects to be implemented on foreign soil carries a host of additional intricacies. Most importantly, the risk embodied in the discounting process should relate to the realization of the discounted cash flows by providers of capital. In cases involving foreign laws, the risk of realizing cash flows (generated by the project) by the investors could be significantly higher than the risk of intrinsic generation of cash flows by the project.

Yet, perhaps the biggest challenge to experts is presented in the valuation of 'intangible assets' (Uzma, 2009). With the gradual realization that such assets contribute significantly to the bottomlines of corporates, their valuation for the purposes of recognition and recording on the balance sheets has become an inescapable exercise. Allied to this, is the seriously worrisome aspect of intangibles valuation for accounting regulatory bodies worldwide that such exercises could facilitate manipulations of reported earnings. Allowing too much laxity for corporateurs carries the inevitable risk of perpetrating and, even encouraging, window dressing and corporate frauds. Such a scenario would also provide escape routes for auditors to shun their responsibilities. Amended provisions of FAS 141(R) read with FAS 157 and Concept Paper 7 of the FASB of United States recognize the DCF technique as a pertinent valuation option. In fact, there are several intangibles where DCF constitutes the only practicable methodology. Nevertheless, even the adaptation of this mechanism for intangible valuation is purely at an evolutionary stage. Considerably more empirical testing needs to be done before a universally acceptable framework is developed.

One needs to appreciate here that intangible valuation is intrinsically a complex exercise in view of the blurred nexus between the asset and the stream of income generated by it. Besides, in valuation of such assets, the inputs to the valuation process usually reflect the valuing entity's own assumptions of the attributes of the asset and the valuation is, therefore, least reliable. This is because most intangible assets do not have an active market and consequently, price data for the said asset or similar assets is rarely available. Similarly, cost estimates for constructing the asset are unlikely to provide any reliable inputs to valuation in most cases because such assets (brands, self generated goodwill etc.) get created as a matter of course e.g. due to the company's market standing, efficient supply and distribution network, superior 'perceived' quality, unique product features/manufacturing process etc. (Crane, 2009)

\section{Relative Evaluation of Valuation Methodologies (Lundholm, 2001, Penman, 2001)}

Valuation methods can, generically, be segregated into three broad categories viz. (i) cash flow based methods e.g. discounting of projected dividends/free cash flows; (ii) income based methods e.g. discounting of residual income; (iii) multipliers e.g. Price -Earnings multipliers. In an ideal setting represented by projections embracing an infinite horizon, all the three approaches converge to the same figure so long as we adhere to the "clear surplus relation" i.e. that net income less net dividends account completely for the change in shareholders' equity so that

$$
I(t)-D(t)=E(t)-E(t-1)
$$

and we define "residual income" of an year as net income for the year less a return on the beginning year's equity balance at a rate equal to the cost of equity 
$R I(t)=I(t)-k_{e} E(t-1)$

The equivalence between the "Dividend Discount Model" and the "Residual Income Model" can be easily established by mathematical induction. We have,

$$
\begin{aligned}
& E(0)+\frac{R I(1)}{1+k_{e}}=E(0)+\frac{I(1)-k_{e} E(0)}{1+k_{e}}=\frac{E(0)+I(1)}{1+k_{e}}=\frac{E(1)+D(1)}{1+k_{e}} \\
& E(0)+\frac{R(1)}{1+k_{e}}+\frac{R I(2)}{\left(1+k_{e}\right)^{2}}=E(0)+\frac{I(1)-k_{e} E(0)}{1+k_{e}}+\frac{I(2)-k_{e} E(1)}{\left(1+k_{e}\right)^{2}} \\
& =\frac{D(1)}{1+k_{e}}+\frac{D(2)+E(2)}{\left(1+k_{e}\right)^{2}}
\end{aligned}
$$

Let us assume that

$$
E(0)+\frac{R I(1)}{1+k_{e}}+\ldots+\frac{R I(k)}{\left(1+k_{e}\right)^{k}}=\frac{D(1)}{1+k_{e}}+\ldots+\frac{D(k)+E(k)}{\left(1+k_{e}\right)^{k}},
$$

then

$$
\begin{aligned}
& E(0)+\frac{R(1)}{1+k_{e}}+\ldots+\frac{R(k)}{\left(1+k_{e}\right)^{k}}+\frac{R(k+1)}{\left(1+k_{e}\right)^{k+1}}=\frac{D(1)}{1+k_{e}}+\ldots+\frac{D(k)+E(k)}{\left(1+k_{e}\right)^{k}}+\frac{R(k+1)}{\left(1+k_{e}\right)^{k+1}} \\
& =\frac{D 1)}{1+k_{e}}+\ldots+\frac{D(k)+E(k)}{\left(1+k_{e}\right)^{k}}+\frac{I(k+1)-k_{e} E(k)}{\left(1+k_{e}\right)^{k+1}}=\frac{D(1)}{1+k_{e}}+\ldots+\frac{D(k)}{\left(1+k_{e}\right)^{k}}+\frac{I(k+1)+E(k)}{\left(1+k_{e}\right)^{k+1}} \\
& =\frac{D(1)}{1+k_{e}}+\ldots+\frac{D(k)}{\left(1+k_{e}\right)^{k}}+\frac{D(k+1)+E(k+1)}{\left(1+k_{e}\right)^{k+1}}
\end{aligned}
$$

so

that

identity

$E(0)+\frac{R I(1)}{1+k_{e}}+\ldots+\frac{R I(k)}{\left(1+k_{e}\right)^{k}}=\frac{D(1)}{1+k_{e}}+\ldots+\frac{D(k)+E(k)}{\left(1+k_{e}\right)^{k}}$

holds for all positive integers $k$. Now, if we extend this summation over the entire life of the firm, the final value of $E(k)$ must necessarily be zero since whatever surplus remains for the equity shareholders on liquidation would be distributed to them as the equivalent of final dividend. In other words, of we sum the above series over the entire life of the firm, we must have

$$
E(0)+\frac{R I(1)}{1+k_{e}}+\ldots+\frac{R I(n)}{\left(1+k_{e}\right)^{n}}=\frac{D(1)}{1+k_{e}}+\ldots+\frac{D(n)}{\left(1+k_{e}\right)^{n}}
$$

thereby establishing the equivalence of the two approaches. However, it is considered impracticable to project out the stream of dividends or residual income over the entire life of the firm and usually, in valuing firms, one makes explicit forecasts for a certain number of years and thereafter uses a steady state growth model for computing a terminal value assuming an appropriate steady state growth rate of an infinite stream of dividend flows/residual income

i.e. $\quad P=\sum_{i=1}^{n} \frac{D(i)}{\left(1+k_{e}\right)^{i}}+\frac{D(n+1)}{\left(1+k_{e}\right)^{n}\left(k_{e}-g\right)}$

or

$P=E(0)+\sum_{i=1}^{n} \frac{R I(i)}{\left(1+k_{e}\right)^{i}}+\frac{R I(n+1)}{\left(1+k_{e}\right)^{n}\left(k_{e}-g\right)}$ where

$D(n+1)=D(n)(1+g) \& R I(n+1)=R I(n)(1+g)$. In such a case, we need to exercise care in computing $D(n+1), R I(n+1)$ to ensure equality of the two approaches. Let $g$ be the steady state growth rate of income as well as equity so that $D(n+1)=E(n)-E(n+1)+I(n+1)=E(n)-(1+g)[E(n)-I(n)]$.

Therefore

$$
\sum_{i=1\left(1+k_{e}\right)^{i}}^{n} \frac{\not i i)}{\left(1+k_{e}\right)^{n}\left(k_{e}-g_{\text {thided }}\right)}=E\left(0+\sum_{i=1}^{n} \frac{H(i)}{\left(1+k_{e}\right)^{i}} \frac{E(n)}{\left(1+k_{e}\right)^{n}}+\frac{\not D n+1}{\left(1+k_{e}\right)^{n}\left(k_{e}-g_{\text {thided }}\right)}\right.
$$

$$
=E(0)+\sum_{i=1}^{n} \frac{R I(i)}{\left(1+k_{e}\right)^{i}}-\frac{E(n)}{\left(1+k_{e}\right)^{n}}+\frac{E(n)-(1+g)[E(n)-I(n)]}{\left(1+k_{e}\right)^{n}\left(k_{e}-g\right)}
$$

$$
=E(0)+\sum_{i=1}^{n} \frac{R I(i)}{\left(1+k_{e}\right)^{i}}-\frac{E(n)}{\left(1+k_{e}\right)^{n}}+\frac{(1+g) I(n)-g E(n)}{\left(1+k_{e}\right)^{n}\left(k_{e}-g\right)}
$$

$=E(0)+\sum_{i=1}^{n} \frac{R(i)}{\left(1+k_{e}\right)^{i}} \frac{E(n)}{\left(1+k_{e}\right)^{n}}+\frac{(1+g) I(n)-k_{e} E(n)+\left(k_{e}-g\right) E(n)}{\left(1+k_{e}\right)^{n}\left(k_{e}-g\right)}$

$=E(0)+\sum_{i=1}^{n} \frac{R\left(1+k_{e}\right)^{i}}{i} \frac{(1+g) I(n)-k_{e} E(n)}{\left(1+k_{e}\right)^{n}\left(k_{e}-g\right)}=E(0)+\sum_{i=1\left(1+k_{e}\right)^{i}}^{n} \frac{R(i)}{\left(1+k_{e}\right)^{n}\left(k_{e}-g\right)}$ 
thus, establishing equality of the two approaches once again. What needs to be noted, however, in the above is that, unlike the usual practice, $D(n+1) \neq D(n)(1+g)$ and $R I(n+1) \neq R I(n)(1+g)$.

It, therefore, follows that the very existence of these differing schemes is mandated more by practical considerations than the underlying philosophy viz. that the enterprise value is the aggregate present value of the entire stream of dividends emanating from the enterprise discounted at the cost of equity. The fact, then, that these methods do, in practice, yield varying outcomes leads us to question the implementation methodology rather than the theoretical premises.

Use of Price -Earnings or other similar multiples of analogous companies for the corporate valuation envisaged are becoming increasingly popular probably because of their perceived simplicity e.g. one develops one's own estimate of the EPS on a set of premises and multiplies it with the P/E multiple averaged over a set of similar companies to arrive at a value of the company's share. However, such use of multipliers comes with several caveats e.g.

(a) The choice of multiples must be consistent with the underlying earnings stream that one is attempting to capitalize. For instance, the $\mathrm{P} / \mathrm{E}$ ratio relates the value of equity to the income stream available to the equity shareholders i.e. profit after tax and preference dividend. It is clearly unsuitable for an exercise which envisages the valuation of a corporate on the free cash flow model. In such a scenario, it would be more desirable to use a multiple based on EBDIT.

(b) A very serious impediment to the use of income based multipliers is that such multipliers are dependent on accounting policies pursued by the relevant corporate e.g. adoption of different methods of depreciation, amortizations of intangibles and their valuations, accounting for foreign exchange transactions and similar accounting issues for which dichotomous or even polychotomous treatment is enabled by the accounting regulators.

(c) Such multipliers are also subject to misevaluations by the markets due to short term aberrations, information asymmetries etc.
There are also a number of computational constraints that limit the use of multipliers for valuation e.g. identifying corporates of similar operational and financing dimensions, existence of similar timing conventions for accounting periods etc.

In today's environment, it is not uncommon to encounter valuation of financial entities or products that have a complex structure of cash flows e.g. projects may have embedded abandonment options, fixed income securities may possess callable or puttable options, convertible shares and/or warrants are, obviously, very well known. In such cases of valuation, one needs to take care of these singular characteristics and ascribe an appropriate figure to the value addition generated there from. A possible approach to this value imputation could be through the use of Black-Scholes option price formula or variants thereof (Singh, 2007). It needs to be emphasized that all pricing models for contingent claims carry with them a bucket of rigid assumptions (e.g. the Black Scholes model imputes a lognormal distribution of the underlying asset price process) so that while adopting any such framework, one should necessarily test the valuation environment against these premises before proceeding with use of the model.

\section{CONCLUSION:}

In this article, we have espoused the cause of DCF valuation emphasizing its immense versatility - this method can be adapted to resolve most valuation problems. However, while the approach has sound underpinnings, its actual implementation warrants care and restraint. Some of the conscientious issues have been attended to in the preceding paragraphs. A deep examination would, nevertheless, reveal that most of the perceived shortcomings of the DCF methodology, in actual fact, owe their origin to faulty implementation and DCF, viewed in itself, makes strong financial sense. To reiterate, a "due diligence" on a DCF exercise needs to focus on the following facets:

(a) That inflation effects are duly incorporated in the analysis i.e. either real cash flows are discounted at real rates or nominal cash flows at nominal rates, we must ensure compatibility between the numerator \& denominator;

(b) The discount rate should be adjusted only for the systematic (market) risk, diversifiable risk 
should carry no weightage insofar as projected returns are concerned;

Appropriate treatment of taxation is paramount because, in most cases of valuation, only the after tax flows are relevant as they constitute the realizable cash flows for the providers of capital;

(d)

Care should be taken to quantify all incremental costs and benefits, whether tangible or otherwise, that are related to or emanate from the asset to be valued;

(e) A related issue is the possible existence of special features embedded in the investment opportunity. A quantification of such attributes is mandatory for a correct application of DCF.

In view of the immense significance of issues of asset valuation in the contemporary environment, they are occupying centre stage across the globe with academicians and practitioners grappling with facts, figures, accounts and mathematics in efforts to achieve a precise valuation model. A school of these workers are attempting to juxtapose probabilistic concepts like expected cash flows together with the probability distribution thereof into the DCF formulation in an attempt to enhance its efficacy (Schumann, 2006). Variants of DCF in the domain of "fuzzy mathematics" have also been propounded (Huang, 2008) . However, it still remains to be seen as to what extent "man can emulate reality".

\section{REFERENCES}

Anderson, Ronald C, Byers, Steven \& Groth, John C, Management Decision, 6, 38, 384, 2000;

Crane, Matthew \& Dyson, Robert A, The CPA Journal, 50, January 2009;

Elton, Edwin J \& Gruber, Martin J, Modern Portfolio Theory \& Investment Analysis, John Wiley, 1997;

Fabozzi, Frank J, Professional Perspectives on Fixed Income Portfolio Management, John Wiley, 2002;
Fernandez, Pablo, managerial Finance, 33, 11, 853, 2007;

Feynmann, R P \& Hibbs, A R, Quantum Mechanics \& Path Integrals, McGraw Hill, 1965;

Golub, Bennett W \& Tilman, Leo M, Risk Management Approaches for Fixed Income Securities, John Wiley, 2000;

Harris, R S \& Pringle, J J, Journal of Financial Research, 237, Fall 1985;

Huang, Xiaoxia, Computers \& Industrial Engineering, 55, 1, 34, August 2008;

Lundholm, Russell \& O'Keefe, Terry, Contemporary Accounting Research, 18, 2, 311, 2001 and references therein;

Martin, P C et al, Phy. Rev. A, 8, 423, 1973;

Miller, M H, Journal of Finance, 261, May 1977;

Modigliani, F \& Miller, M, American Economic Review, 48, 261, 1958;

Myers, S C, Journal of Finance, 1, March 1974;

Penman, Stephen H, Graduate School of Business, Columbia Universite website, August 2001 and refernces therein;

Revuz, Daniel \& Yor, Marc, Continuous Martingales \& Brownian Motion, Springer, 1999:

Salomon Brothers, Understanding the Yield Curve, United States Fixed-Income Research Portfolio Strategies, September 1995;

Schumann, S, Valuation Strategies, 10, 1, Sep/Oct 2006;

Singh J P \& Prabakaran S, Electronic $\mathrm{J}$ of Theoretical Physics, 3, 13, 19, 2006;

Singh $\mathrm{J} P$ \& Prabakaran S, Electronic $\mathrm{J}$ of Theoretical Physics, 4, 14, 69, 2007;

Tuckman, Bruce, Fixed Income Securities - Tools for Today's Markets, John Wiley, 2002;

Uzma S H \& Singh J P, Chartered Secretary, August, 2009; Vasicek, O A, J Financial Economics, 5, 177, 1977;

Wilmott, Paul, Quantitative Finance, John Wiley, 2000; 